\title{
Wash-Free Redox Cycling Based Electrochemical Biosensors for Point-of-Care Diagnostic Applications
}

\author{
Gorachand Dutta* \\ Department of Electronic \& Electrical Engineering, University of Bath, UK
}

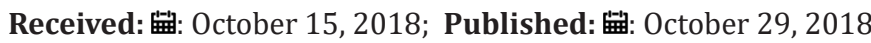

*Corresponding author: Gorachand Dutta, Centre for Biosensors, Bioelectronics and Biodevices (C3Bio), Department of Electronic \& Electrical Engineering, University of Bath, Bath BA2 7AY, UK

\begin{abstract}
The main driving force of point-of-care testing require to bring the test methods conveniently and immediately to the patient. This is particularly true for the developing countries with less developed health care infrastructure. However, a simple and reliable biosensing technique to an affordable platform is often challenging [1-3]. Mostly, Enzyme-labels are used in electrochemical biosensors for signal amplification [4]. The enzyme-based biosensors are most famous because of its high and reproducible signal amplification [5]. But there is a big question mark for the stability of an enzyme and not suitable for bed-side applications. Also, another problem for the enzyme is the direct electron transfer between enzyme label and electrode is a formidable challenge because of the large electron-hoping distance between the electrode and the redox center of the enzyme label [6]. As a result, the signal amplification by enzymatic reaction is not suitable for the early stage diseases detection. Redox cycling is a process that can help to overcome this limitation by repetitively generate or consume signaling species (molecules or electrons) in the presence of reversible redox specie [7]. Many redox cycling processes can be combined with biosensor for the ultrasensitive biomarkers detection i.e. electrochemicalelectrochemical (EE) redox cycling, electrochemical-chemical (EC) redox cycling, chemical-chemical (CC) redox cycling or electrochemical-chemicalchemical (ECC) [3,8].
\end{abstract}

A combination of redox cycling and electrochemical detection can play a significant role for the early stage diseases detection. Electrochemical biosensor technique is most popular and ideal technique for point-of-site application because of their low cost, high sensitivity, portable fieldbased size, and rapid diagnosis [9-11]. However, it is extremely challenging to originate an electrochemical point-of-site technique retaining both simplicity and very high sensitivity. There has been an increased attempt towards the development of electrochemical redox cycling techniques to develop the disposable rapid test for early stage cancer and infectious diseases biomarkers detection for point-of-care diagnosis [12-15]. Currently many biosensors using affinity binding between antigen and antibody have been developed but most of them have a drawback in terms of simplicity, rapidness, cost-effectiveness and ultra-sensitivity [16-18]. Most reported biosensors need many steps washing before the actual sensing measurement and that's why those immunoassays are not applicable for bed side application. If a wash-free electrochemical scheme is combined with the assays, this could significantly simplify the detection procedure and reduce the assay time [19-21]. In this review wash-free redox cycling technologies are focused on for simple, cost-effective and portable immunosensors that can be operated for the applications in bed-side diagnostics.

Keywords: Redox Cycling; Electrochemical; Immunosensors; Wash Free; Point of Care; Lab on Chip

Abbreviations: EE: Electrochemical-Electrochemical; EC: Electrochemical-Chemical; CC: chemical-chemical; ECC: Electrochemical-ChemicalChemical; EN: Electrochemical-Enzymatic; CPE: $\pi$-Conjugated Polyelectrolyte

\section{Mini Review}

\section{Wash-Free Redox Cycling Techniques}

Washing-free redox cycling immunoassay technique was developed by Dutta et al. [22,23] to minimize the washing steps of the immunoassay that allows fast, sensitive, and single-step detection of biomarkers in serum with low interference. Electrochemicalenzymatic (EN) redox cycling (Figure1) was used to amplify the signal-to-background ratios. Biotinylated capture probe (IgG) was immobilized on the ITO electrode surface. A sample solution (contains unknown concentration of biomarker) was prepared with enzyme-conjugated IgG and enzyme-substrate, which was spiked with serum with different concentrations of target antigen. The solution mixture was then injected into the electrochemical cell and incubated for $10 \mathrm{~min}$. The interference effect was minimized by applying a lower applied potential and eliminating the ascorbic acid effect. A calibration plot was obtained with increasing the target concentration. The signal was increased with the target concentration because surface concentration of bound enzyme- 
conjugated IgG was increased with increase in target concentration. The surface bound enzyme allowed faster electron mediation than an unbound enzyme. The limit of detection (LoD) was $1 \mathrm{pg} / \mathrm{mL}$ in PBS and $10 \mathrm{pg} / \mathrm{mL}$ in serum for PSA.

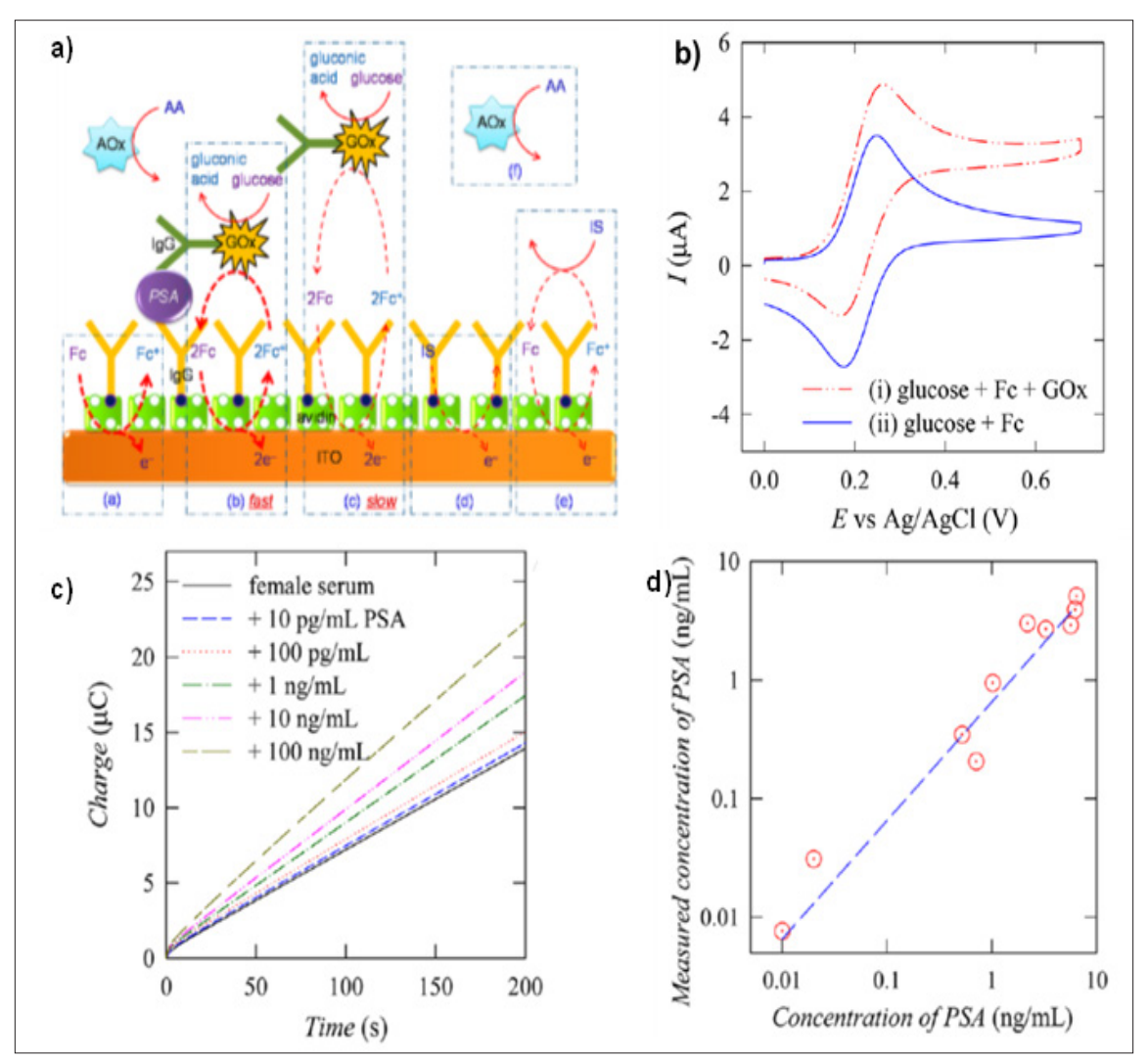

Figure 1: (a) Schematic diagram of a washing-free immunosensor using proximity-dependent electron mediation (b) Cyclic voltammograms for (i) PBS containing 5.0mM glucose, $100 \mu \mathrm{M} \mathrm{Fc}$, and 100 $\mu \mathrm{g} / \mathrm{mL}$ GOx, (ii) PBS containing 5.0mM glucose and $100 \mu \mathrm{M} \mathrm{Fc}$, (c) Chronocoulogram recorded at immunosensing electrodes for detecting different PSA concentrations in real samples. (d) A comparison graph between washing-free immunosensor and a commercial instrument (Reprinted with permission from Dutta et al. 2014. Copyright (2014) American Chemical Society).

An electrochemical-enzymatic redox cycling and wash-free technique was presented by Nandhakumar et al. [24] to detect cortisol where a competitive displacement method was used in human serum. The electrochemical signal was mainly contributed by the bound conjugate than the unbound one and the detection limit was $\sim 30 \mathrm{pM}$ within $12 \mathrm{~min}$. The developed wash-free sensor can be used for simple, sensitive, and rapid point-of-care diagnosis of small molecules. An electrochemical enzymatic redox cyclingbased wash-free DNA detection protocol was reported by Fang et al. [25] using proximity-dependent electron mediation. This wash-free technique could discriminate between target template DNA of Piscirickettsia salmonis and nontarget DNAs using a Zinc Finger Protein. The detection limit was approximately 300 copies in $13.2 \mu \mathrm{L}$, indicating an ultrasensitive detection method. An electrochemical-chemical (EC) redox cycling-based wash-free DNA sensor mediated by Conjugated Polyelectrolyte was reported by Park et al. [26] An anionic $\pi$-conjugated polyelectrolyte (CPE) label having many redox-active sites showed faster electron mediation after sandwich-type target-specific binding. The fast CPE-mediated oxidation of ammonia borane along the entire CPE backbone (EC redox cycling) affords high signal amplification and avoid the washing steps for biomarkers detection.

\section{Conclusion}

In this review, a simple and cost-effective wash-free redox cycling detection method was discussed for point-of-care testing such as medical diagnostics, biological research, environmental monitoring and food analysis. This simple technique can help to develop portable diagnostic biodevices which is urgently required for the developing countries with less developed health care infrastructure. In future, printing technology on flexible substrate and wash-free method could open new opportunities for the development of bioelectronics toward practical applications. Furthermore, the real sample analysis in the wash-free chip will make the diagnostic process highly applicable for bed side application.

\section{Acknowledgement}

Dr. Gorachand Dutta gratefully acknowledges to Dr. Despina Moschou for her support in University of Bath, UK and Professor 
Haesik Yang for his guidance during my $\mathrm{PhD}$ in the wash-free sensors.

\section{References}

1. Gubala V, Harris LF, Ricco AJ, Tan MX, Williams DE (2012) Point of care diagnostics: status and future. Anal Chem 84(2): 487-515.

2. Jia Y, Sun H, Li X, Sun D, Hu T, et al. (2018) Paper-based graphene oxide biosensor coupled with smartphone for the quantification of glucose in oral fluid. Biomed Microdevices 20(4): 89.

3. Dutta G, Rainbow J, Zupancic U, Papamatthaiou S, Estrela P, et al. (2018) Microfluidic Devices for Label-Free DNA Detection. Chemosensors 6(4): 43.

4. Grieshaber D, MacKenzie R, Voros J, Reimhult E (2008) Electrochemical Biosensors - Sensor Principles and Architectures. Sensors 8(3): 14001458 .

5. Yang H (2012) Enzyme-based ultrasensitive electrochemical biosensors. Current Opinion in Chemical Biology 16(3-4): 422-428.

6. Bourdillon C, Demaille C, Moiroux J, Savéant J M (1996) From Homogeneous Electroenzymatic Kinetics to Antigen-Antibody Construction and Characterization of Spatially Ordered Catalytic Enzyme Assemblies on Electrodes. Acc Chem Res 29(11): 529-535.

7. Akanda MR, Choe YL, Yang H (2012) Outer-sphere to inner-sphere" redox cycling for ultrasensitive immunosensors. Anal Chem 84(2): 1049-1055.

8. (2017) Electrochemical Redox Cycling Amplification Technology for Point-of-Care Cancer Diagnosis.

9. El Harrad L, Bourais I, Mohammadi H, Amine A (2018) Recent Advances in Electrochemical Biosensors Based on Enzyme Inhibition for Clinical and Pharmaceutical Applications. Sensors 18(1).

10. Farzin L, Shamsipur M, Samandari L, Sheibani S (2018) Recent advances in designing nanomaterial based biointerfaces for electrochemical biosensing cardiovascular biomarkers. J Pharm Biomed Anal 161: 344376.

11. Mishra GK, Barfidokht A, Tehrani F, Mishra RK (2018) Food Safety Analysis Using Electrochemical Biosensors. Foods 7(9).

12. Singh A, Park S, Yang H (2013) Glucose-Oxidase Label-Based Redox Cycling for an Incubation Period-Free Electrochemical Immunosensor. Anal Chem 85(10): 4863-4868.

13. Akanda MR, Joung HA, Tamilavan V, Park S, Kim S, et al. (2014) An interference-free and rapid electrochemical lateral-flow immunoassay for one-step ultrasensitive detection with serum. Analyst 139(6): 14201425

\section{ISSN: 2574-1241}

DOI: $10.26717 /$ BJSTR.2018.10.001962

Gorachand Dutta. Biomed J Sci \& Tech Res

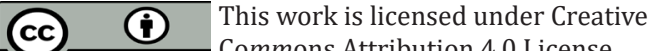

Submission Link: https://biomedres.us/submit-manuscript.php
14. Dutta G, Lillehoj PB(2017) An ultrasensitive enzyme-free electrochemical immunosensor based on redox cycling amplification using methylene blue. Analyst 142(18): 3492-3499.

15. Dutta G, Nagarajan S, Lapidus LJ, Lillehoj PB (2017) Enzyme-free electrochemical immunosensor based on methylene blue and the electro-oxidation of hydrazine on Pt nanoparticles. Biosens Bioelectron 92: 372-377.

16. Capoferri D, Della Pelle F, Del Carlo M, Compagnone D (2018) Affinity Sensing Strategies for the Detection of Pesticides in Food. Foods 7(9).

17. Hasanzadeh M, Sahmani R, Solhi E, Mokhtarzadeh A, Shadjou N, et al. (2018) Ultrasensitive immunoassay of carcinoma antigen 125 in untreated human plasma samples using gold nanoparticles with flower like morphology: A new platform in early stage diagnosis of ovarian cancer and efficient management. Int J Biol Macromol 119: 913-925.

18. Kuss S, Amin HMA, Compton RG (2018) Electrochemical Detection of Pathogenic Bacteria-Recent Strategies, Advances and Challenges. Chem Asian J 13(19): 2758-2769.

19. Kaisti M, Kerko A, Aarikka E, Saviranta P, Boeva Z, et al. (2017) Real-time wash-free detection of unlabeled PNA-DNA hybridization using discrete FET sensor. Sci Rep 7(1): 15734.

20. White RJ, Kallewaard HM, Hsieh W, Patterson AS, Kasehagen JB, et al. (2012) Wash-free, electrochemical platform for the quantitative, multiplexed detection of specific antibodies. Anal Chem 84(2): 10981103.

21. Bechstein DJ, Lee JR, Ooi CC, Gani AW, Kim K, et al. (2015) High performance wash-free magnetic bioassays through microfluidically enhanced particle specificity. Sci Rep 5: 11693.

22. Dutta G, Kim S, Park S, Yang H (2014) Washing-Free Heterogeneous Immunosensor Using Proximity-Dependent Electron Mediation between an Enzyme Label and an Electrode. Anal Chem 86(9): 4589-4595.

23. Dutta G, Park S, Singh A, Seo J, Kim S, et al. (2015) Low-Interference Washing-Free Electrochemical Immunosensor Using Glycerol-3phosphate Dehydrogenase as an Enzyme Label. Anal Chem 87(7): 35743578.

24. Nandhakumar P, Haque AJ, Lee NS, Yoon YH, Yang H (2018) Washing-Free Displacement Immunosensor for Cortisol in Human Serum Containing Numerous Interfering Species. Anal Chem 90(18): 10982-10989.

25. Fang CS, Kim KS, Ha DT, Kim MS, Yang H (2018) Washing-Free Electrochemical Detection of Amplified Double-Stranded DNAs Using a Zinc Finger Protein. Anal Chem 90(7): 4776-4782.

26. Park S, Jeong JE, Le VS, Seo J, Yu B, et al. (2018) Enhanced Electron Transfer Mediated by Conjugated Polyelectrolyte and Its Application to Washing-Free DNA Detection. J Am Chem Soc 140(7): 2409-2412.

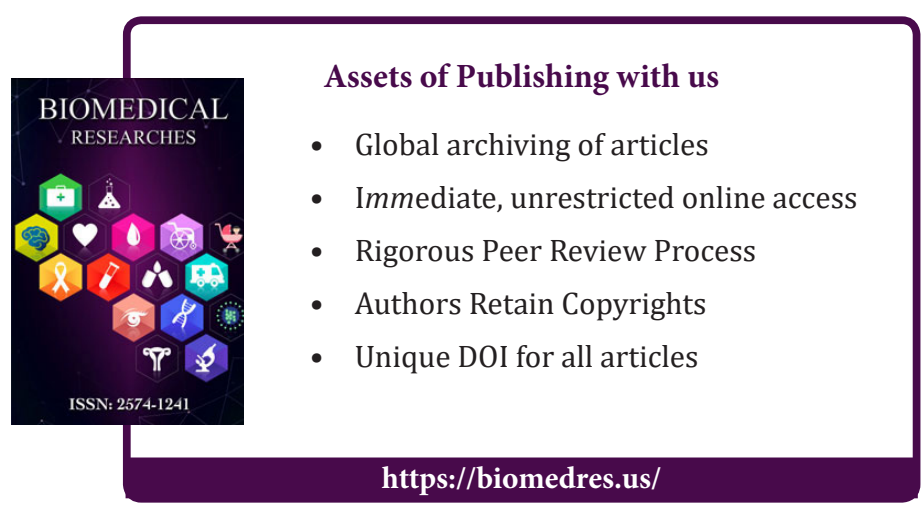

Cite this article: Gorachand Dutta.Wash-Free Redox Cycling Based Electrochemical Biosensors for Point-of-Care Diagnostic Applications. 